\title{
A Simultaneous Discover-Identify Approach to Causal Inference in Linear Models
}

\author{
Chi Zhang, ${ }^{1}$ Bryant Chen, ${ }^{2}$ Judea Pearl ${ }^{1}$ \\ ${ }^{1}$ Department of Computer Science, University of California, Los Angeles, California, USA \\ ${ }^{2}$ Brex, San Francisco, California, USA* \\ \{zccc, judea\}@cs.ucla.edu, bryant@brex.com
}

\begin{abstract}
Modern causal analysis involves two major tasks, discovery and identification. The first aims to learn a causal structure compatible with the available data, the second leverages that structure to estimate causal effects. Rather than performing the two tasks in tandem, as is usually done in the literature, we propose a symbiotic approach in which the two are performed simultaneously for mutual benefit; information gained through identification helps causal discovery and vice versa. This approach enables the usage of Verma constraints, which remain dormant in constraint-based methods of discovery, and permit us to learn more complete structures, hence identify a larger set of causal effects than previously achievable with standard methods.
\end{abstract}

\section{Introduction}

Learning causal relationships is one of the most ambitious goals of scientific inquiry. Controlled randomized experiments can sometimes be used to both learn the causal structure among variables, as well as the size of the causal effects. However, such experiments are often too expensive or even impossible to conduct. Instead, learning causal relationships from observational data can be attempted; first by learning the causal structure from observational data, called discovery, and then identifying causal effects from the observational data and the partially specified causal structure. This paper introduces a method of performing both tasks simultaneously in a mutually beneficial way.

Many algorithms have been developed for causal discovery. These algorithms generally fall into two categories: score-based algorithms (e.g., Heckerman, Geiger, and Chickering (1995), Chickering (2002), Shpitser et al. (2012), Fast GES by Ramsey et al. (2017)) and constraintbased algorithms (e.g., IC algorithm by Verma and Pearl (1991), PC algorithm by Spirtes et al. (2000), FCI algorithm first by Spirtes et al. (2000) and improved by Zhang (2008)). Constraint-based algorithms aim to discover a class of graphs that encode the same constraints as those implied

\footnotetext{
*Much of the work by Chen was conducted while at IBM Research AI.

Copyright (C) 2020, Association for the Advancement of Artificial Intelligence (www.aaai.org). All rights reserved.
}

(a)

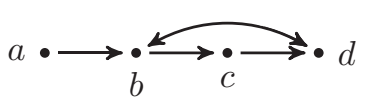

Figure 1: (a) a DAG where $\sigma_{a d} / \sigma_{a c}=\sigma_{c d \cdot b}$ (b) a DAG where $\sigma_{a d} / \sigma_{a c} \neq \sigma_{c d \cdot b}$

by the data. They perform a sequence of conditional independence tests to efficiently rule out impossible edge configurations. Constraint-based algorithms have significant advantage over score-based algorithms in that they are able to learn entire equivalence classes of models with unobserved variables, often called "semi-Markovian."

Existing constraint-based algorithms use conditional independences between model variables to learn the causal structure. However, since there are usually many structures consistent with any given set of conditional independences, these algorithms are only able to produce large equivalence classes of possible structures.

Verma constraints (Verma and Pearl 1991) impose additional constraints on the probability distribution beyond conditional independences, and thus allow the discovery of additional structures. For example, though Figures 1(a) and 1(b) are conditional-independence-equivalent, they imply different Verma constraints. 1(a) implies the Verma constraint $\sigma_{a d} / \sigma_{a c}=\sigma_{c d \cdot b}$, while 1(b) does not (hint: $\sigma_{a d}$ is equal to the product of the three coefficients on $a \rightarrow b, b \rightarrow c$, and $c \rightarrow d$ in 1(a), while $\sigma_{a d}$ is equal to the same product plus the coefficient on $a \rightarrow d$ in 1(b)). Several algorithms for deriving Verma constraints from a model's structure have been developed, including algorithms by Tian and Pearl (2002) and Shpitser and Pearl (2008) for non-parametric models and algorithms by Chen (2016) and Chen, Kumor, and Bareinboim (2017) for linear models. These algorithms can be used to derive Verma constraints from a hypothesized model structure and test it. However, it is not clear how to systematically find such constraints from data to discover the model's structure. Indeed, no constraint-based method for learning causal structures from Verma constraints currently exists in the literature. 
Fortunately, under the linear setting, a useful tool, called auxiliary variables (AVs) (Chen, Pearl, and Bareinboim 2015), can be used to reduce the problem of finding Verma constraints to one of finding conditional independences. AVs are constructed by subtracting known direct effects-if the coefficient from variables $x$ to $y, \beta$, is known, an AV $y^{*}=y-\beta x$ is constructed by subtracting $\beta x$ from $y$. Now, $y^{*}$ may be conditionally independent of some variables that $y$ was dependent of. This conditional independence, which is equivalent to a Verma constraint over the original model variables, can then be used to learn more of the structure.

Constructing AVs without prior knowledge requires identification of direct effects. Thus, in order to use AVs in causal discovery, we need a method to identify direct effects from an incomplete causal structure. To this end, we generalize the qID algorithm of Chen, Kumor, and Bareinboim (2017) for partially specified causal structures. Combining this algorithm with AVs, we are able to iteratively identify causal effects on an incomplete structure, construct AVs, and learn more of the structure. Each identification step enables the construction of more AVs, which helps to learn more of the structure. Similarly, each causal discovery step learns more of the structure, which helps to identify more causal effects.

In summary, we introduce a simultaneous discoveridentify algorithm, where each task is performed to the other's benefit. To our knowledge, this algorithm is the first constraint-based causal discovery algorithm to use Verma constraints, and the first identification algorithm for partially specified linear causal models ${ }^{1}$. Lastly, we demonstrate that in high percentages of simulated cases, our method provides noticeable improvements in recovering random graph structures while guaranteeing correctness.

\section{Preliminaries}

The causal directed acyclic graph (DAG) of a structural equation model (SEM) is a graph, $G=(V, E)$, where $V$ are nodes representing model variables and $E$ are edges representing causal relations between two nodes. An edge in a causal graph can be directed $(\rightarrow)$, bidirected $(\leftrightarrow)$, or both. Directed edges encode the direction of causality, i.e., if $x_{i}$ is in the structural equation that determines $x_{j}$, an edge is drawn from $x_{i}$ to $x_{j}$. Each directed edge, therefore, is associated with a coefficient in the SEM, which we often refer to as its edge coefficient. A bidirected edge between two nodes indicates their corresponding error terms may be statistically dependent, while the lack of a bidirected edge indicates the error terms are independent. If both a directed edge and a bidirected edge exist between two nodes, it indicates one variable is directly affecting the other and they are both affected by an unobserved confounder at the same time.

In the following sections, we use standard graph terminology, where $\mathrm{He}(E)$ denotes the heads of a set of directed edges, $E, T a(E)$ denotes the tails, and for a node $v$, the set of edges for which $\mathrm{He}(E)=v$ is denoted $\operatorname{Inc}(v)$. We also restrict our attention to semi-Markovian linear causal mod-

\footnotetext{
${ }^{1}$ Non-parametric algorithms can, of course, also be applied to linear models, but they are significantly weaker due to their inability to leverage the linearity assumption.
}

els (Pearl 2009), models that are acyclic, that may contain latent confounders, and for which the causal relationships are linear. Lastly, we use the term full $D A G^{2}$ to refer to a standard causal graph, where the orientation of every edge is specified, and the term true DAG to refer to the full DAG that represents the underlying data generating process.

We use $\sigma_{x y \cdot W}$ to denote the partial covariance between two variables, $x$ and $y$, given a set of variables, $W$. We also assume without loss of generality that the model variables have been standardized to mean 0 and variance 1 .

\section{Patterns}

When learning a causal structure, constraints on the covariances between variables (conditional independence and Verma constraints) are generally insufficient to define a single DAG. Instead, they are only able to narrow down the set of possible structures to a large equivalence class. Patterns are motivated by the need to define a graph structure to represent such a class. Using causal discovery algorithms, we aim to learn a pattern that represents an equivalence class of graphs consistent with the constraints provided.

Similar concepts were previously defined in the literature, including patterns in Verma and Pearl (1991) (who first used the term "pattern") and partial ancestral graphs (PAGs) in Richardson (1996). PAGs are used to represent equivalence classes of maximal ancestral graphs (MAGs) (Richardson and Spirtes 2002). MAGs are abstractions of DAGs that keep only the conditional independence and ancestral relationships. More formally, MAGs are maximal and ancestral. There is an edge between two nodes $a$ and $b$ in the MAG if and only if there exists no set that can separate $a$ and $b$ in the DAG (maximal), and $a \rightarrow b$ is in the MAG if and only if $a$ is an ancestor of $b$ in the DAG (ancestral).

PAGs are useful for causal discovery algorithms such as FCI, which aims to recover a MAG. However, PAGs cannot distinguish between different DAGs sharing the same MAG abstraction, and therefore cannot distinguish between different DAGs sharing ancestral relationships and conditional independence constraints but have different Verma constraints. For example, in Figure 2(a), $e$ and $f$ are not conditionally independent. Therefore, a DAG with $e$ and $f$ connected and a DAG without them connected share the same MAG, even though they imply different Verma constraints. Since our method will enable us to distinguish between such structures, we need a more precise representation without the "maximal" or "ancestral" requirement.

Definition 1. A pattern, $P=(V, E)$, is a graph whose edges contain three possible types of edge marks: arrowheads, tails, and circles (and hence four kinds of edges ${ }^{34}$ :

\footnotetext{
${ }^{2}$ We emphasize a DAG being "full" to distinguish it from a "pattern", which is a partially specified DAG. Note that we are not referring to a complete DAG, which is a DAG where all edges are present.

${ }^{3}$ These edge markings are adopted from PAGs.

${ }^{4}$ We assume no selection bias. The other two kinds of edges in PAGs defined in Zhang (2008), - and o-, which only appear when there is selection bias, are thus not included.
} 
(a)

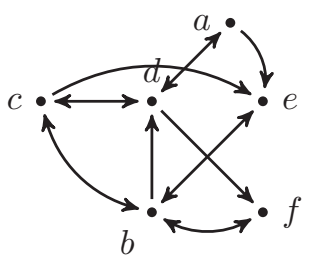

(c)

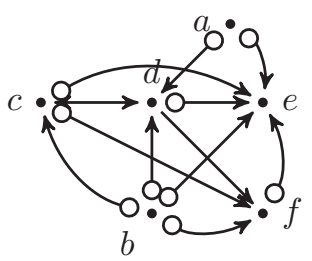

(b)

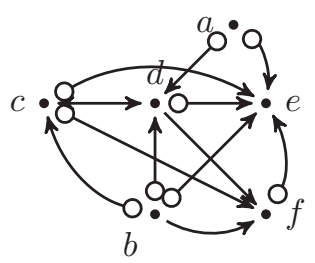

(d)

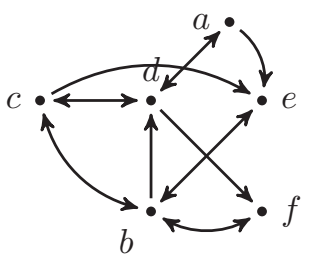

Figure 2: (a) underlying causal relationships (b) pattern learned by FCI (c) pattern learned by modified FCI, which does not learn inconsistent tails such as $b \rightarrow f$ in (b) (d) pattern learned by our method, LCDI

$\rightarrow, \leftrightarrow, \circ-\circ, \circ \rightarrow)$. The edges denote possible causal relations between two nodes.

Each pattern $P$ can be used to represent (formally defined below) a class of full DAGs, denoted $[G]$. A circle mark indicates uncertainty, i.e., it is possible that the edge mark is arrowhead for some members in $[G]$, tail for some members, and both (having both a directed edge and a bidirected edge in between) for others. An edge mark is said to be invariant if the mark is the same in all members of $[G]$ (Zhang 2008).

Definition 2. A pattern $P=\left\{V_{P}, E_{P}\right\}$ is defined to represent a class of full DAGs [G], if for each member $G=$ $\left\{V_{G}, E_{G}\right\}$ in $[G]$, (i) $V_{P}=V_{G}$, and (ii) each e $\in E_{P}$ is either extraneous (the two same nodes in $G$ are not connected by an edge), or the arrowhead and tail edge marks on e are invariant in $[G]$.

In Figure 2, 2(a) is both in the class represented by 2(c) and the class represented by $2(\mathrm{~d})$. This is seen by checking each edge. For example, $a \circ \rightarrow d$ in 2(c) has an arrowhead at $d$ and a circle at $a$, so the DAGs in the class it represents must have an arrowhead at $d$ but can have anything at $a . a \leftrightarrow d$ in 2(a) satisfies the requirement. $e \leftarrow \circ f$ in 2(c) is extraneous since it is not in 2(a), which also satisfies Definition 2. Note that from a causal discovery perspective, learning 2(d) is preferable to learning 2(b) since the class of graphs represented by 2(d) is a subset of the class represented by 2(b).

\section{Edge Orientation Rules Based on Verma Constraints}

In this section, we first review how conditional independence constraints are used by current causal discovery algorithms before describing how we extend these algorithms by incorporating Verma constraints. First, conditional independence constraints are found by checking the partial cor- (a)
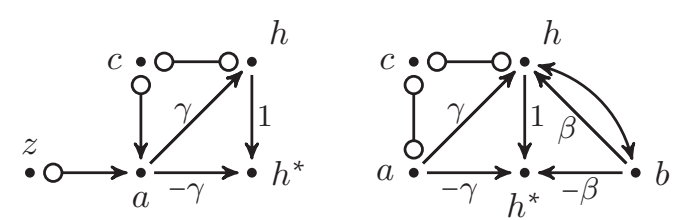

Figure 3: (a) an AV in a pattern (b) an AV generated by two variables in a pattern

relation between each pair of variables given all subsets of other variables. Assuming faithfulness, each vanishing partial correlation indicates there is no edge between the pair of variables, and the conditioning set contains the variables that, when conditioned on, d-separates the pair in the graph. Therefore, we are able to rule out the edge orientations that leave an unblocked path between the pair.

Current constraint-based causal discovery methods use only conditional independence constraints because conditional independence constraints can be easily found, and their implications on the structure is clear. In contrast, Verma constraints are hard to find without the aid of a full DAG, because their functional forms are far less restricted. Additionally, it is also not always clear how they constrain the graph structure.

However, by identifying causal effects and constructing $\mathrm{AVs}$, we may generate new conditional independences between the AVs and the original model variables. These conditional independences, which we describe as AV conditional independence constraints, are Verma constraints. Thus, by using AVs, we can reduce the problem of finding and using Verma constraints for causal discovery to a problem of finding and using conditional independences-a problem that is already well understood.

Intuitively, AVs negate the effect of problematic paths by subtracting out known direct effects. Let $P^{E+}$ denote the augmented pattern with AVs generated using edges $E$ added. In Figure 3(a), if the direct effect of $a$ on $h, \gamma$, is identified, an $\mathrm{AV}, h^{*}=h-\gamma a$ can be generated, giving $P^{a h+}$. Similarly, in Figure 3(b), an AV $h^{*}=h-\gamma a-\beta b$ can be generated using edges $a \rightarrow h, b \rightarrow h$, giving $P^{\{a h, b h\}+}$. Generating AVs from patterns will allow us to search the data for new conditional independences involving the AVs and learn more of the model's structure. These conditional independences correspond to Verma constraints over the original model variables as explained in the following lemma.

Lemma 1. Given an $A V, z^{*}=z-\Sigma_{i} e_{i} t_{i}$, the conditional independence constraint, $\sigma_{a z^{*} \cdot S}=0$, is equivalent to the Verma constraint, $\sigma_{a z \cdot S}-\Sigma_{i} e_{i} \sigma_{a t_{i} \cdot S}=0$, where $S$ is a set of variables. Furthermore, this Verma constraint cannot, in general, be represented as a conditional independence constraint over the original model variables, $V$.

Lemma 1 makes it possible to easily find Verma constraints that are AV conditional independence constraints ${ }^{5}$.

\footnotetext{
${ }^{5}$ There might exist other types of Verma constraints that cannot
} 
We can simply check whether each AV can be made conditionally independent of other AVs or the original model variables. Similar to traditional conditional independence constraints, AV conditional independence constraints refine the structure by limiting edge marks to those that block all the paths between the independent variables in the augmented pattern. Furthermore, this is in fact equivalent to blocking paths in the pattern without the edges used to generate the AVs, as stated in the following corollary, derived from Theorem 1 in Chen, Kumor, and Bareinboim (2017).

Corollary 1. Given a linear pattern $P$ representing [G], where $E \subset \operatorname{Inc}(z)$ is a set of edges whose coefficient values are known, if $(W \cup\{y\}) \cap\left(V \backslash N D e^{*}(z)\right)=\varnothing$, and $G_{E-}$ represents the graph $G$ with the edges for $E$ removed, then $\sigma_{z^{*} y \cdot W}=0$ only if $(z \Perp y \mid W)_{G_{E-}}$ for all $G$ in $[G]$.

See the pattern $P^{\{a \rightarrow h\}+}$ in Figure 3(a), where the edge coefficient on $a \rightarrow h, \gamma$, is identified (using $z$ as an instrumental variable) and the $\mathrm{AV}, h^{*}=h-a \gamma$, is constructed. If $\exists S_{a h^{*}}, \sigma_{a h^{*} \cdot S_{a h^{*}}}=0$, then Corollary 1 implies for all $G$

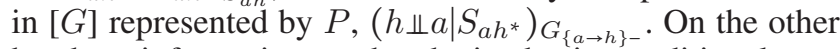
hand, no information can be obtained using traditional conditional independence constraints. $\nexists S_{a h}, a \Perp h \mid S_{a h}$ since $a$ and $h$ are directly connected by an edge.

Assuming a generalized version of faithfulness ${ }^{6}$, the only path between $a$ and $h$ in $G_{\{a \rightarrow h\}_{-}}, a \leftarrow \circ c \circ-\circ h$, must be blocked by $S_{a h^{*}}$. If, for example, $c \notin S_{a h^{*}}, c$ must be a collider in any $G$, and we can thus orient $a \leftrightarrow c \leftarrow \circ$ in $P$.

To formally construct the edge orientation rules, we need to characterize such a relationship between two variables like $a$ and $h$ that are not necessarily non-adjacent in the original pattern, but are non-adjacent in $P_{E-}$ due to the independence between their AVs. We also need to characterize variables remaining to be adjacent in $P_{E-}$ such that the adjacencies of all variables are with respect to the same pattern, with or without $E$ virtually removed, to ensure consistent edge orientations. We describe such adjacency relationships in the following definition.

Definition 3. Given an AV-augmented pattern $P^{E+}$ where $A V s, a^{*}=a-\sum_{i} e_{a i} t_{a i}$ and $b^{*}=b-\sum_{j} e_{b j} t_{b j}$, are generated, and $E=\left\{e_{a i}\right\}_{i} \cup\left\{e_{b j}\right\}_{j}$ is the set of all edges subtracted to construct $a^{*}$ and $b^{*}$. $a$ and $b$ are generalized adjacent in $P^{E+}$, denoted $\operatorname{adj}_{E}(a, b)$, if $\nexists S, \sigma_{a^{*} b^{*} \cdot S}=0$. Otherwise, $a$ and $b$ are generalized non-adjacent in $P^{E+}$, denoted $\operatorname{nadj}_{E}(a, b)$. We denote the set $S$ where $\sigma_{a^{*} b^{*} \cdot S}=0$ as $S_{a b}^{*}$.

A special case of Definition 3 is when only one $\mathrm{AV}, b^{*}$, is generated, i.e., $a d j_{E}(a, b)$ if $\nexists S, \sigma_{a b^{*} \cdot S}=0$, and $\operatorname{nadj}_{E}(a, b)$ otherwise. Next, we generalize discriminating path given in Zhang (2008), which is necessary for constructing one of the edge orientation rules. See Figure 4 for a graphical illustration.

be expressed as AV conditional independence constraints. Those constraints are outside the scope of this paper.

${ }^{6}$ Typically, faithfulness implies that path-separation (Pearl 2009) in the true DAG precisely characterizes conditional independence in the data distribution. In our case, we require a slightly stronger version of this assumption in which Theorem 1 of Chen, Kumor, and Bareinboim (2017) precisely characterizes the AVconditional independence constraints in the data.

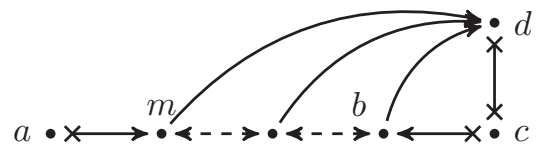

Figure 4: A generalized discriminating path, $u=$ $\langle a, m, \cdots, b, c, d\rangle$, between $a$ and $d$ for $c$

Definition 4 (generalized discriminating path). $u=$ $\langle a, \cdots, b, c, d\rangle$, is a generalized discriminating path between $a$ and $d$ for $c$ if

(i) $u$ includes at least three edges;

(ii) $c$ is a non-end node on $u$, and is adjacent to $d$ on $u$;

(iii) every node between $a$ and $c$ is a collider on $u$ and is a parent of $d$; and

(iv) denote $m$ as the node following $a$ on $u$ (can be b). $\exists E \in E_{a d}, \operatorname{nadj}_{E}(a, d), a_{d j}(a, m)$, and for every node $n$ between $a$ and $d, \operatorname{adj}_{E}(n, d)$.

Now, we construct the edge orientation rules based on AV conditional independence constraints. These rules generalize the rules of the FCI algorithm for DAGs for generalized adjacency and non-adjacency and are iteratively performed. $E_{K}$ denotes the set of known or identified directed edges at the current iteration. For simplicity, for each pair of variables $a$ and $b$, we define $E_{a b}=\left\{E_{K} \cap \operatorname{Inc}(a), E_{K} \cap \operatorname{Inc}(b), E_{K} \cap\right.$ $(\operatorname{Inc}(a) \cup \operatorname{Inc}(b))\}$. The edge mark $*$ is a wildcard representing any of an arrowhead, a tail, and a circle, and remains the same after an orientation rule.

Rule 0: For every adjacent pair $a$ and $b$, if $\exists E \in E_{a b}$, $\operatorname{nadj}_{E}(a, b)$, and the edge $a *-* b$ is not in $E$, record $a *-* b$ as extraneous without removing it.

Rule 1: For every triple $a, b$ and $c$, if (i) $\exists E \in E_{a c}$, $\operatorname{nadj}_{E}(a, c), a d j_{E}(a, b), a d j_{E}(b, c)$, and (ii) $b \notin S_{a c}^{*}$, then orient $a * \rightarrow b \leftarrow * c$.

Rule 2: For every triple $a, b$ and $c$, if (i) $\exists E \in E_{a c}$, $\operatorname{nadj}_{E}(a, c), a d j_{E}(a, b), a d j_{E}(b, c)$, (ii) $b \in S_{a c}^{*}$, and (iii) $a * \rightarrow b \circ-* c$, then orient $a * \rightarrow b \rightarrow c$.

Rule 3: For every pair $a$ and $d$, if $\exists u=\langle a, \cdots, b, c, d\rangle$, a generalized discriminating path between $a$ and $d$ for $c$, then

(i) if $c \notin S_{a d}^{*}$, orient $b \leftrightarrow c \leftarrow * d$,

(ii) if $c \in S_{a d}^{*}, b \leftrightarrow c$, and $c \circ-* d$, orient $c \rightarrow d$,

(iii) if $c \in S_{a d}^{*}, d \leftrightarrow c$, and $c \circ \rightarrow b$, orient $c \rightarrow b$.

Rules 0-3 describe how to use AV conditional independences found in the data to orient edges. Rule 0 is a special case of blocking paths. An edge in a pattern $P$ is regarded extraneous with respect to the true DAG $G$ if the two nodes on that edge in $P$ are non-adjacent in $G$. Consider the example in Figure 2. Figure 2(a) is the true DAG. Figure 2(b) is the pattern learned using the FCI algorithm, where only traditional conditional independence constraints are used. Extraneous edges $c \circ \rightarrow f, d \circ \rightarrow e$, and $e \leftarrow \circ f$ that do not exist in the true DAG are learned, because there is no separating set $W$ for $c$ and $f$ such that $c \Perp f \mid W$, and same for the other two pairs. However, we do not remove the extraneous 
edge, $a *-* b$, immediately when it is found. This is because when performing other orientation rules, if $a d j_{E}(a, b)$ for that $E$, then $a *-* b$ can be used the same way as if it were non-extraneous, which might help orient other edges.

Rule 1 states that $b$ must be a collider if $a$ and $c$ are independent without conditioning on $b$ but dependent when conditioning on $b$. Rule 2 states that the middle node cannot be a collider if $a$ and $c$ are independent when conditioning on $b$ but dependent otherwise. The example of Figure 3(a) explained before is an application of Rule 1.

Rule 3 is more complicated. The intuition behind discriminating paths is to choose orientations for $b \leftarrow * c$ and $c *-* d$ that block the paths between $a$ and $d$. If $a$ and $d$ are nonadjacent, there exists a conditioning set, $S$, that blocks all the paths between them. All the nodes between $a$ and $d$ on $u$ must be in $S$, because otherwise there is an unblocked path $a * \rightarrow m \leftrightarrow-\cdots \rightarrow d$. Therefore, $u$ must be unblocked from $a$ to $c$, and we have to block $u$ at $c$. Now, we just have to check if $c \in S$, and $b \leftarrow * c$ and $c *-* d$ can be oriented the same ways as Rules 1 and 2, where part (i) in Rule 3 corresponds to Rule 1 and parts (ii) and (iii) in Rule 3 correspond to Rule 2. Compared to the original definition of discriminating paths, generalized discriminating paths do not require $a$ and $d$ to be non-adjacent, but only require them to be generalized non-adjacent, and all the adjacent nodes to be generalized adjacent. Changing those adjacency relationships to generalized adjacencies can be understood as virtually removing $E$ in order to analyze the paths between those nodes in $P_{E-\text {. }}$.

\section{Causal Identification in Patterns}

Generating AVs requires either a priori knowledge of coefficient values or identification of coefficients. In this section, we show how to identify causal effects in linear patterns, which will allow us to use AVs to help learn causal structures from obserevational data. For example, in Figure 2(c), the edge $d \rightarrow f$ is identifiable using the instrumental variable (IV) method (Bowden and Turkington 1990). Although the DAG is incomplete, we can still see there is no unblocked path between $a$ and $f$ not through $d$ (we can see this by enumerating all possibilities of circle marks), which makes $a$ a valid IV. In other words, for any full DAG represented by this pattern, the coefficient on $d \rightarrow f$ is equal to $\sigma_{a f} / \sigma_{a d}$.

The most general, efficient ${ }^{7}$ identification algorithm in fully specified linear SCMs is the qID method (Chen, $\mathrm{Ku}$ mor, and Bareinboim 2017). qID uses quasi-instrumental sets, which are an extension of generalized instrumental sets (Brito and Pearl 2002) for AVs. Our method can be understood as defining a stricter version of quasi-instrumental set for patterns, named determinate quasi-instrumental set. More formally, if $Z$ is a determinate quasi-instrumental set for edges $E$ in a pattern $P$, then $Z$ is a quasi-instrumental set for $E$ in any member of $[G]$ represented by $P$. This will enable us to identify $E$ given $P$, and is guaranteed to give the same results as if we had the true DAG $G_{t r u e}$, as long as $G_{\text {true }}$ belongs to $[G]$.

\footnotetext{
${ }^{7} \mathrm{qID}$ is polynomial-time if the degree of the nodes are bounded.
}

To achieve this goal, we first define determinate descendants $\left(D e^{*}\right)$, determinately unblocked paths, determinate non-descendants $\left(N D e^{*}\right)$, determinately blocked paths, and determinately $d$-separated $\left(d s e p^{*}\right) . y$ is a determinate descendant $\left(D e^{*}\right)$ of $x$ in pattern $P$, if $x$ is a descendant of $y$ in every graph represented by $P$. Similarly, $p$ is a determinately unblocked path in $P$ if it is an unblocked path in all graphs represented by $P$. Determinate non-descendant, determinately blocked path, and determinately d-separated are similarly defined. Lastly, a set of paths have no sided intersection if for every pair of paths, they do not share any node that has an arrow to the same direction on both paths (Foygel et al. 2012). Characterizations for each of these definitions in patterns are given in the Appendix.

Now, we describe how to find determinate quasiinstrumental sets in a pattern.

Theorem 1. Given a linear SEM with pattern $P$, a set of edges $E_{K}$ whose coefficient values are known, and a set of structural coefficients $\alpha=\left\{\alpha_{1}, \alpha_{2}, \cdots, \alpha_{k}\right\}$, the set $Z=\left\{z_{1}, \cdots, z_{k}\right\}$ is a determinate quasi-instrumental set for $\alpha$ if there exist triples $\left(z_{1}, W_{1}, \pi_{1}\right), \cdots,\left(z_{k}, W_{k}, \pi_{k}\right)$ such that:

(i) For $i=1, \cdots, k$, either:

(a) $W_{i} \in N D e^{*}(y)$, and $d \operatorname{sep}^{*}\left(z_{i}, W_{i}, y\right)_{P_{E \cup E_{y^{-}}}}$where $E_{y}=E_{K} \cap \operatorname{Inc}(y)$, or

(b) $W_{i} \in N D e^{*}(y) \cap N D e^{*}\left(z_{i}\right)$, and dsep $^{*}\left(z_{i}, W_{i}, y\right)_{P_{E \cup E_{z y^{-}}}}$where $E_{z y}=E_{K} \cap(\operatorname{Inc}(z) \cup$ $\operatorname{Inc}(y))$

(ii) for $i=1, \cdots, k, \pi_{i}$ is a path between $z_{i}$ and $x_{i}$ that is determinately unblocked by $W_{i}$ in $P_{E \cup E_{y}-}$ if $z_{i}$ satisfies $(i)(a)$ and in $P_{E \cup E_{z y}-}$ if $z_{i}$ satisfies $(i)(b)$, where $x_{i}=\operatorname{Ta}\left(\alpha_{i}\right)$, and

(iii) the paths $\left\{\pi_{1}, \cdots, \pi_{k}\right\}$ have no sided intersection.

Theorem 2 (Identifiability). If $Z$ is a determinate quasiinstrumental set for $E$, then $E$ is identifiable.

In addition to enabling the usage of AVs and, therefore, the usage of Verma constraints in causal discovery, identification in patterns is also useful on its own. It allows us to compute causal effects from incomplete or even zero knowledge about the underlying causal structure.

\section{Algorithm for Learning Patterns and Identification}

In this section, we construct an algorithm for simultaneous causal discovery and identification. When learning a pattern from data and prior knowledge, we want the pattern to contain only features in the true DAG, but also be as specific as possible, i.e., we want to learn as many invariant arrowheads and tails as possible and remove as many extraneous edges as possible. As we have discussed, structure learning and causal identification can benefit each other. Learning a more precise pattern helps with identifying more edges. Identifying more edges allows us to create more AVs and learn more AV conditional independence constraints, which helps with learning a more precise structure. We construct the Linear Causal Discovery and Identification (LCDI) 
algorithm that implements this bootstrapping procedure to learn a pattern $P$ and identify causal coefficients given observational data.

\section{Linear Causal Discovery and Identification (LCDI)}

Input: covariance matrix $\sigma_{V}$ on the set of observed variables $V$ and a set of identified edges $E_{i d}$ (can be empty)

Output: a pattern $P$ and updated $E_{i d}$

Step 0: Run FCI algorithm (Zhang 2008) on $\sigma_{V}$ with Rules $\mathcal{R} 1-\mathcal{R} 4$ only, but replacing $\mathcal{R} 4$ with $\mathcal{R} 4$ - given below. The resulting pattern is $P$;

Step 1: Run the original FCI algorithm on $\sigma_{V}$ with Rules $\mathcal{R} 1-\mathcal{R} 4$ and $\mathcal{R} 8-\mathcal{R} 10^{8}$ to obtain a PAG $P^{\prime}$, and merge the arrowheads in $P^{\prime}$ to $P$;

Step 2: Repeat the following Substeps on $P$ until neither $P$ nor $E_{i d}$ is updating;

Substep 0: Perform causal identification on $P$ without extraneous edges and update $E_{i d}$;

Substep 1: Generate AVs using $E_{i d}$;

Substep 2: Run Rules 0-3;

Substep 3: Run FCI algorithm $\mathcal{R} 1$ and $\mathcal{R} 4+$ (given below) repeatedly until $P$ is not updating;

Step 3: Remove all the extraneous edges marked in Rule 0 in Step 1 Substep 2 from $P$.

$\mathcal{R} 4-$ and $\mathcal{R} 4+$ below are modified from FCI. $S_{a d}$ denotes the set of conditioning variables which makes $a$ and $d$ independent.

$\mathcal{R} 4-: \quad u=\langle a, \cdots, b, c, d\rangle$ is a discriminating path $^{9}$ between $a$ and $d$ for $c$; then

(i) if $c \notin S_{a d}$, and $c \circ-* d$, orient $b \leftrightarrow c \leftrightarrow d$;

(ii) if $c \in S_{a d}$, and $c *-\circ d$, orient $c * \rightarrow d$.

$\mathcal{R} 4+: \quad u=\langle a, \cdots, b, c, d\rangle$ is a discriminating path between $a$ and $d$ for $c$; then

(i) if $c \notin S_{a d}$, orient $b \leftrightarrow c \leftarrow * d$ if not done so;

(ii) if $c \in S_{a d}, b \leftrightarrow c$, and $c \circ-* d$, orient $c \rightarrow d$;

(iii) if $c \in S_{a d}, d \leftrightarrow c$, and $c \circ \rightarrow b$, orient $c \rightarrow b$;

(iv) if $c \in S_{a d}$, and $c *-\circ d$, orient $c * \rightarrow d$.

We use $\mathcal{R} 4-$ and $\mathcal{R} 4+$ instead of $\mathcal{R} 4$ because FCI tries to recover the MAG representation for the true DAG, while our method aims to recover the true DAG directly. They make sure the resulting pattern is consistent with the true DAG instead of the MAG. We skip the tail orientation rules $\mathcal{R} 8-\mathcal{R} 10$ in the original FCI for the same reason. See the next section for a more detailed discussion of MAGs and DAGs. The correctness of LCDI is summarized in the following theorem.

Theorem 3. $P$ is the pattern output by LCDI, then the true $D A G G$ that was used to generate the covariance matrix $\sigma_{V}$ must be a member of $[G]$ represented by $P$.

\footnotetext{
${ }^{8}$ We skip $\mathcal{R} 5$ - $\mathcal{R} 7$ because they are useful in dealing with selection bias, while we assume no selection bias.

${ }^{9} \mathrm{~A}$ discriminating path is defined as a generalized discriminating path replacing all generalized adjacency relationships with normal adjacency relationships in Definition 4.
}

Theorem 3 shows that any arrowhead or tail learned by LCDI must be present in the true DAG. Algorithms such as FCI that aim to recover a MAG only guarantees tail correctness regarding the MAG converted from the true DAG, but might learn tails that do not exist in the true DAG. However, correct tail orientations are an important factor for causal inference since they help distinguish between direct causation and confounded correlation, while LCDI guarantees tail soundness regarding the true DAG.

We will use the example of Figure 2 to illustrate LCDI. Figure 2(a) shows the underlying true DAG we want to recover. LCDI begins with Step 0, an iteration of modified FCI, which utilizes conditional independence constraints to learn the pattern in Figure 2(c). Extraneous edges $c \circ \rightarrow f$, $d \circ \rightarrow e$, and $e \leftarrow \circ f$ are learned, because there is no separating set that can make each pair of variables conditionally independent. In Step 1, we merge the arrowheads from the PAG learned using FCI, shown in 2(a), to the pattern from Step 0. In this specific example, no arrowhead is newly added. However, there are cases where FCI learns additional arrowheads that cannot be learned using modified FCI.

Next, in Step 2 Substep 0, the only identifiable edge in Figure 2(c) is $d \rightarrow f$, using $\{a\}$ as a determinate quasiinstrumental set. This allows the $\mathrm{AV}, f^{*}=f-a \cdot \alpha$, where $\alpha$ is the coefficient on $d \rightarrow f$, to be generated in Substep 1. Next, in Substep 2, LCDI searches for conditional independences between the newly generated AVs and other variables. In Rule $0, \operatorname{nadj}_{\{d \rightarrow f\}}(c, f)$ since $\sigma_{c f^{*} \cdot \varnothing}=0$, and $c \circ \rightarrow f$ is recorded as extraneous. Similarly, $e \leftarrow \circ f$ is recorded as extraneous. In Rule 1, $\operatorname{nadj}_{\{d \rightarrow f\}}(c, f)$ and $b \notin S_{c f}^{*}$ give orientations $c \leftrightarrow b \leftrightarrow f$, and $n a d j_{\{e \rightarrow f\}}(c, f)$ and $b \notin S_{c f}^{*}$ give orientation $e \leftrightarrow b$. In Rule 3, we can find a generalized discriminating path, $u=\langle c, d, b, f\rangle$ between $c$ and $f$ for $b$, and condition (iii) gives $b \rightarrow d$.

In the next iteration of Step 2, we find $b \rightarrow d$ is now identifiable using $\{b\}$ as a determinate quasi-instrumental set, and as before, $d \circ \rightarrow e$ is marked extraneous. In Step 2, we orient $d \leftrightarrow a \leftrightarrow e, c \rightarrow e$, and $e \leftrightarrow f$.

In the third iteration of Step 2, we find $c \rightarrow e$ is identifiable using $\{c\}$ as a determinate quasi-instrumental set. No more edge orientations can be deduced. Lastly, in Step 3, all the three extraneous edges are removed, and we obtain the final pattern, Figure 2(d).

Compared to the pattern learned by FCI in Figure 2(b), the pattern learned by LCDI was much more informative. First, LCDI removed all the extraneous edges, while FCI had three of them. Second, LCDI learned more edge orientations (in this specific example, LCDI was even able to recover all the edge orientations!) while FCI had quite a few circle marks. Third, LCDI guaranteed tail soundness regarding the true DAG, while FCI oriented $b \rightarrow f$, which was in the MAG representation of the true DAG, but was inconsistent with the true DAG itself.

The runtime of LCDI is composed of two parts, identification and structure update. Denote the runtime of qID in (Chen, Kumor, and Bareinboim 2017) as $q$, the runtime of FCI in (Zhang 2008) as $f$, the number of iterations run as $r$, then the runtime of LCDI is $\mathcal{O}(r(q+f)) . r$ is bounded by 


\begin{tabular}{|c|cccccc|}
\hline$d$ & 6 & 7 & 8 & 9 & 10 & 11 \\
\hline$(1.5,2]$ & 1.0 & 4.0 & 2.0 & 1.0 & 1.5 & 2.0 \\
\hline$(2.75,3.25]$ & 5.5 & 8.0 & 15.5 & 18.5 & 23.5 & 25.5 \\
\hline$(4,4.5]$ & 0 & 8.0 & 15.0 & 32.5 & 36.5 & 45.0 \\
\hline
\end{tabular}

Table 1: percentage of graphs where LCDI learns more arrowheads than FCI

\begin{tabular}{|c|cccccc|}
\hline$d$ & 6 & 7 & 8 & 9 & 10 & 11 \\
\hline$(1.5,2]$ & 17.4 & 19.2 & 13.0 & 12.5 & 13.8 & 11.6 \\
\hline$(2.75,3.25]$ & 11.1 & 8.7 & 12.7 & 9.6 & 10.4 & 7.4 \\
\hline$(4,4.5]$ & 0 & 8.7 & 7.4 & 8.0 & 7.0 & 6.8 \\
\hline
\end{tabular}

Table 2: percentage more of arrowheads LCDI learns than FCI in graphs where LCDI learns more arrowheads

the number of edges in the initial pattern, but is likely to be much smaller.

\section{Simulation Results}

To illustrate the advantages of LCDI, we compare it with FCI, which is considered to be the current state of the art constraint-based causal discovery algorithm without additional assumptions on the data distribution. FCI was first proposed by Spirtes et al. (2000), and the improved version by Zhang (2008) achieved arrowhead and tail completeness, i.e., it can learn every invariant arrowhead and tail for the equivalence class of MAGs. However, FCI might recover more tails than there are in the true DAG, because the MAG itself might have more tails. The PAG in Figure 2(b) has a directed edge, $b \rightarrow f$, which is in fact a bidirected edge, $b \leftrightarrow f$, in the true DAG (2(a)). However, FCI does not recover more arrowheads than there are in the true DAG. The following theorem shows the power of orienting arrowheads in LCDI.

Theorem 4. Under the linear setting and given the covariance matrix of the data, if an invariant arrowhead can be recovered by $F C I$, then it can be recovered by $L C D I$.

Theorem 4 results directly from how LCDI is constructed, and it implies that LCDI always recovers equal or more correct arrowheads compared to FCI.

To quantify this improvement, we implemented LCDI and the version of FCI by Zhang (2008). We randomly generate DAGs with number of nodes $(n)$ from 6 to 11 with various average node degrees $(d)$, and an edge being directed and bidirected both have probability 0.5 . We then compare the patterns that would be learned on the generated DAG by each method assuming faithfulness. More specifically, we compare the number of invariant arrowheads and extraneous edges learned. Each data entry in Tables 1 and 2 was averaged over 200 random DAGs.

Table 1 shows for DAGs of different node numbers, the percentages of DAGs where LCDI learns at least one more arrowhead than FCI, for different $d$ ranges $((1.5,2]$, $(2.75,3.25],(4,4.5])$. As we can see, the benefit of LCDI generally increases with the number of nodes in the DAG. In
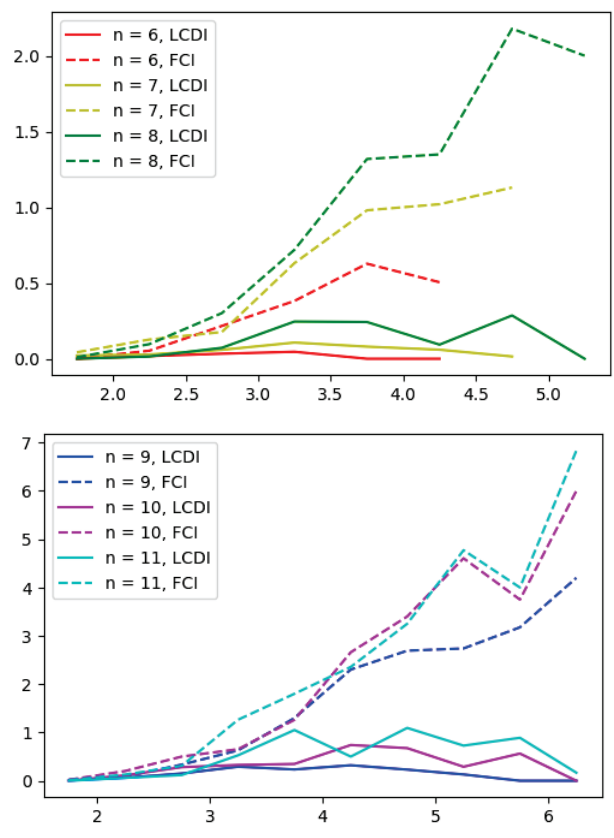

Figure 5: numbers of extraneous edges learned by FCI vs. numbers of extraneous edges learned by LCDI

over $45 \%$ of the DAGs with $n=11$ and large $d$, LCDI learns more arrowheads, which is a significant improvement.

Table 2 shows for the DAGs where LCDI learns more arrowheads, how much more can LCDI learn compared to FCI. For any $n$, it can recover $10 \%$ to $20 \%$ of total arrowheads more than FCI when $d$ is small.

Figure 5 shows the numbers of extraneous edges learned by FCI and LCDI. The different colors indicate DAGs of different node numbers. On average, LCDI learns less than 1 extraneous edge for any $n$ and $d$, while the number of extraneous edges FCI learns increases as $n$ and $d$ increases.

We can see LCDI provides decent improvements in a large percentage of random DAGs-it learns more arrowheads and less extraneous edges. Furthermore, these improvements do not sacrifice correctness. All the arrowheads and tails LCDI learns and all the extraneous edges it removes are guaranteed to be in the true DAG.

\section{Related Work}

Shpitser, Richardson, and Robins (2009) introduced a method to test extraneous edges using Verma constraints under the non-parametric setting. Their work is limited to full DAGs and is not generalized to partial DAGs.

Jaber, Zhang, and Bareinboim (2018) introduced an identification method for PAGs. Their method works in the nonparametric setting. In comparison, our method can identify some causal effects that cannot be identified without assuming linearity. In addition, our method is applied to patterns, which are consistent with the true DAG.

Shpitser et al. (2012) introduced a score-based causal discovery method. Their method incorporates Verma constraints in a different way: their Q-FIT algorithm fits pa- 
rameters such that if two graphs are equivalent in terms of Verma constraints, they have the same score. Their method searches for graphs with highest likelihood score based on data. However, the resulting graph is a full DAG. Therefore, even though that DAG is Verma-constraint-equivalent to the true DAG, we still might not be able to infer what structures the true DAG has, since it is in general impossible to list all equivalent DAGs and summarize their characteristics. In comparison, our method is constraint-based, and learns an equivalent class that is guaranteed to represent the true DAG.

Shimizu et al. (2006) introduced a linear causal discovery method. It assumes non-faithfulness, no latent confounders, and non-Gaussian errors. In contrast, we assume faithfulness and relax the other two assumptions.

\section{Conclusion}

In this paper, we developed a symbiotic approach to causal discovery and identification in linear models. We first formally defined the type of partially specified DAGs, patterns, that are useful for both causal discovery and identification. We then devised a method of incorporating Verma constraints using auxiliary variables, and method of identification on patterns. Finally, we developed an algorithm that performs causal discovery and identification simultaneously, for mutual benefit. We showed that the combined algorithm performs better than doing each task separately. In addition, our algorithm can learn more complete structures than previously reported algorithms.

\section{Acknowledgements}

Zhang and Pearl are supported in parts by grants from International Business Machines Corporation (IBM) [\#A1771928], National Science Foundation [\#IIS-1527490 and \#IIS1704932], and Office of Naval Research [\#N0001417-S-B001]. The authors would like to thank Yujia Shen, Elias Bareinboim, and Carlos Cinelli for helpful discussions.

\section{References}

Bowden, R. J., and Turkington, D. A. 1990. Instrumental variables, volume 8. Cambridge University Press.

Brito, C., and Pearl, J. 2002. Generalized instrumental variables. In Proceedings of the Eighteenth conference on Uncertainty in artificial intelligence, 85-93. Morgan Kaufmann Publishers Inc.

Chen, B.; Kumor, D.; and Bareinboim, E. 2017. Identification and model testing in linear structural equation models using auxiliary variables. In Proceedings of the 34th International Conference on Machine Learning-Volume 70, 757766. JMLR. org.

Chen, B.; Pearl, J.; and Bareinboim, E. 2015. Incorporating knowledge into structural equation models using auxiliary variables. arXiv preprint arXiv:1511.02995.

Chen, B. 2016. Identification and overidentification of linear structural equation models. In Lee, D. D.; Sugiyama, M.; Luxburg, U. V.; Guyon, I.; and Garnett, R., eds., Advances in Neural Information Processing Systems 29. Curran Associates, Inc. 1579-1587.
Chickering, D. M. 2002. Optimal structure identification with greedy search. Journal of machine learning research 3(Nov):507-554.

Foygel, R.; Draisma, J.; Drton, M.; et al. 2012. Half-trek criterion for generic identifiability of linear structural equation models. The Annals of Statistics 40(3):1682-1713.

Heckerman, D.; Geiger, D.; and Chickering, D. M. 1995. Learning bayesian networks: The combination of knowledge and statistical data. Machine learning 20(3):197-243.

Jaber, A.; Zhang, J.; and Bareinboim, E. 2018. Causal identification under markov equivalence. arXiv preprint arXiv:1812.06209.

Pearl, J. 2009. Causality. Cambridge university press.

Ramsey, J.; Glymour, M.; Sanchez-Romero, R.; and Glymour, C. 2017. A million variables and more: the fast greedy equivalence search algorithm for learning high-dimensional graphical causal models, with an application to functional magnetic resonance images. International Journal of Data Science and Analytics 3(2):121-129.

Richardson, T., and Spirtes, P. 2002. Ancestral graph markov models. Ann. Statist. 30(4):962-1030.

Richardson, T. 1996. A discovery algorithm for directed cyclic graphs. In Proceedings of the Twelfth international conference on Uncertainty in artificial intelligence, 454461. Morgan Kaufmann Publishers Inc.

Shimizu, S.; Hoyer, P. O.; Hyvärinen, A.; and Kerminen, A. 2006. A linear non-gaussian acyclic model for causal discovery. Journal of Machine Learning Research 7(Oct):2003-2030.

Shpitser, I., and Pearl, J. 2008. Dormant independence. Technical Report R-340L, $<$ http://ftp.cs.ucla.edu/pub/stat_ser/r340-L.pdf $>$, Department of Computer Science, University of California, Los Angeles, CA. Extended version of paper that appeared in AAAI-08.

Shpitser, I.; Richardson, T. S.; Robins, J. M.; and Evans, R. 2012. Parameter and structure learning in nested markov models. arXiv preprint arXiv:1207.5058.

Shpitser, I.; Richardson, T. S.; and Robins, J. M. 2009. Testing edges by truncations. In Twenty-First International Joint Conference on Artificial Intelligence.

Spirtes, P.; Glymour, C. N.; Scheines, R.; Heckerman, D.; Meek, C.; Cooper, G.; and Richardson, T. 2000. Causation, prediction, and search. MIT press.

Tian, J., and Pearl, J. 2002. On the testable implications of causal models with hidden variables. In Proceedings of the Eighteenth conference on Uncertainty in artificial intelligence, 519-527. Morgan Kaufmann Publishers Inc.

Verma, T., and Pearl, J. 1991. Equivalence and synthesis of causal models. UCLA, Computer Science Department.

Zhang, J. 2008. On the completeness of orientation rules for causal discovery in the presence of latent confounders and selection bias. Artificial Intelligence 172(16-17):18731896. 